\title{
2-hydroxylated sphingomyelin profiles in cells from patients with mutated fatty acid 2-hydroxylase
}

\author{
Phyllis Dan', Simon Edvardson², Jacek Bielawski ${ }^{3}$, Hiroko Hama ${ }^{3}$ and Ann Saada ${ }^{\text {* }}$
}

\begin{abstract}
Fatty acid 2-hydroxylase (FA2H) is the enzyme responsible for the hydroxylation of free fatty acids prior to their incorporation into 2-hydroxylated sphingolipids, which are the major constituents of the myelin leaflet. Mutated FA2H has been associated with neurodegenerative diseases. Decreased FA2H activity was demonstrated only in vitro, but not in patient tissues. In this study we characterized the 2-hydroxylated sphingomyelin (SM) profiles in blood and fibroblasts from patients harboring a deleterious FA2H mutatation, and found that hydroxylated fatty acid sphingomyelin is present in normal amounts in patient lymphocytes, but decreased to a different extent in fibroblasts and erythrocytes.
\end{abstract}

Keywords: Fatty acid hydroxylase hydroxylated fatty acid sphingomyelin, autosomal recessive leukodystrophy

\section{Background}

A compact myelin sheet surrounding the axon is essential for correct nerve conduction [1]. Myelin is composed of over $70 \%$ lipids, the most abundant of which are the galactolipids galactosylceramide ( $\mathrm{GalC})$ and its sulfated form, sulfatide. As more than fifty percent of GalC and sulfatide are hydroxylated at the $\mathrm{C} 2$ position on the fatty acid (FA) moiety, it has been estimated that approximately twenty five percent of the outer leaflet lipids in myelin are hydroxylated [2,3]. Based on studies of model membranes, hydroxylation likely contributes to the stability of myelin by virtue of the hydrogen bonding between the hydroxy groups, the galactose head group and the polar part of the ceramide backbone [4].

The 2- hydroxylation of sphingolipids [for review, see [5]] occurs during de novo ceramide synthesis and is catalyzed by the enzyme fatty acid 2-hydroxylase (FA2H), a membrane-bound protein containing a cytochrome b5-like heme-binding domain responsible for the redox activity, and a sterol desaturase domain [6]. The products of FA2H are free 2-hydroxy fatty acids

\footnotetext{
* Correspondence: annsr@hadassah.org.il

'Department of Genetics and Metabolic Diseases and the Monique and Jacques Roboh Department of Genetic Research, Hadassah-Hebrew University Medical Center, Jerusalem, Israel

Full list of author information is available at the end of the article
}

(OHFA), which are subsequently incorporated into ceramide, the precursor of galactosylceramide [7]. FA2Hdeficient mice lacking hydroxylated fatty acids in the central and peripheral nervous system had normal neuronal development, but showed late onset axon and myelin sheet degeneration [8] and exhibited CNS dysfunction [9].

Mutations in the human $F A 2 H$ gene were identified in patients with autosomal recessive leukodystrophy characterized by childhood (4-5 y) onset spasticity, dystonia and white matter degeneration. In seven patients, the desaturase domain was disrupted by a splice site mutation causing the skipping of exons 5 and 6 while a missense mutation in a conserved residue was detected in the other two patients [10]. Recently, mutated FA2H was also found to be the underlying cause of a complicated hereditary spastic paraplegia (SPG35) [11], and neurodegeneration with brain iron accumulation [12]. Although in vitro transfection studies of mutated FA2H disclosed reduced hydroxy fatty acid synthesis, decreased enzymatic activity was not demonstrated in patients. In fact, tetracosanoic acid hydroxylating activity in patient fibroblasts was indistinguishable from that of normal controls [10]. Moreover, the impact of defective FA2H on fatty acid composition in patients is (to our knowledge) unknown. The aim of this study was to 
characterize the functional impact of FA2H splice site mutation on the fatty acid and hydroxy fatty acid sphingomyelin profiles in patient's blood and fibroblasts.

\section{Methods}

Cell culture reagents were obtained from Biological Industries, Beit HaEmek, Israel. Hisopaque and all other reagents were from Sigma-Aldrich, Israel.

\section{Subjects}

Blood from two patients and fibroblasts from one patient harboring the FA2H c.786 $1 \mathrm{G} \rightarrow$ A mutation (family 1, described by Edvardsson et al [10] and from three controls were obtained with informed consent and approval from the local IRB.

\section{Lymhocyte isolation}

Lymphocytes were isolated from whole blood (EDTA anti-coagulant) using Histopaque-1077 according to the manufacturer's instructions. The resulting pellet was lyophilized prior to lipid analysis.

\section{Erythrocyte membrane preparation}

Erythrocyte membranes were prepared from whole blood collected with heparin as anti-coagulant. The blood was spun at $1000 \times \mathrm{g}$ for $10 \mathrm{~min}$, the plasma was removed and the erythrocytes were washed three times with twice their volume of saline. Subsequently, cells were hemolyzed in $5 \mathrm{ml}$ of water then spun at $12000 \times$ $\mathrm{g}$ for 10 minutes. The membranes were washed twice with water and lyophilized prior to lipid analysis.

\section{Cell culture}

Primary fibroblasts were grown in DMEM (high glucose) supplemented with $15 \%$ fetal calf serum in the presence of penicillin and streptomycin. Confluent cells were harvested by trypsinization, washed with phosphate buffered saline (PBS) and lyophilized prior to lipid analysis.

\section{Lipid analysis}

Lipid analysis was performed at the Lipidomics Core of the Medical University of South Carolina using HPLC/ MS-MS as previously described [13,14]. All values are reported normalized to $\mathrm{mg}$ protein as determined using the Lowry method [15].

\section{RT-PCR}

Total RNA from lymphoblasts was prepared using TRI reagent (Sigma Aldrich) and equal amounts were reverse transcribed using ImProm-II (Promega, Wisconsin, USA) reverse transcriptase kit with a hexamer mixture as the template primer according to the manufacturer's instructions. Primer sequences for PCR analysis available upon request.

\section{Results and discussion}

We have previously shown that the c.786+1G $\rightarrow$ A mutation causes mis-splicing leading to skipping of exons 5 and 6 in fibroblasts. RT-PCR was perfomed in order to verify the exon skipping in lymphocytes. The expected 426bp shorter transcript, obtained in the patient, corroborated the exon skipping as shown in Figure 1A.

Subsequently we proceeded to investigate the sphingomyelin (SM) profiles in these cells as well as in erythrocyte membranes and fibroblasts (Figure 1B-C).

The total SM-fatty acid content in patient fibroblasts and lymphocytes was not significantly different from that of control subjects, while patient erythrocytes had a relatively higher SM-fatty acid content (Figure 1B). SMhydroxy fatty acid content in patient lymphocytes was not significantly different from that of the controls, however it was reduced by $50 \%$ in patient fibroblasts and increased in patient erythrocytes (Figure $1 \mathrm{C}$ ). The decrease in SM-hydroxy fatty acid content in fibroblasts was especially evident when the ratio of SM-hydroxy fatty acid to SM-fatty acid was calculated. In patient erythrocytes this ratio was also significantly decreased as SM-hydroxy fatty acids were decreased relative to SMfatty acids Figure 1D).

The distribution of the SM-fatty acid (Figure 2A, B) and SM-hydroxy fatty acids (Figures 2C, 2D) by chain length was investigated. C16 is by far the most abundant fatty acid in all cell types. No differences were seen between patient and control lymphocytes. SM-C16 fatty acid content was slightly increased in patient fibroblasts, and the levels of most SM-fatty acids were increased in patient erythrocytes. In all cell types, in control as well as patient, the distribution of SM-hydroxy fatty acids does not mirror that of their fatty acid counterpart. In lymphocytes, SM-OHC18 is the most abundant species, and the distribution of SM-hydroxy fatty acids is the same in the patient and the control. However, patient fibroblasts contain approximately $50 \%$ of SM-OHC16 and SM-OHC18 of the control values, and all the other SM-hydroxy fatty acid levels were significantly lower. The patient erythrocytes had an elevated content of all SM-hydroxy fatty acids, but with a significant increase of SM-OHC18 vs SM-OHC16.

The fraction of hydroxylated fatty acid relative to fatty acid (OHFA-SM/FA-SM) of the same chain length was calculated (Figure 2E, 2F). This ratio is the same for both control and patient lymphocytes, where the ratio is highest for C18. In contrast, in patient fibroblasts, the fraction of hydroxylated C20:1 is only $1 / 10$ that of the control value, with reductions for $\mathrm{C} 18$ and $\mathrm{C} 20$ as well. 


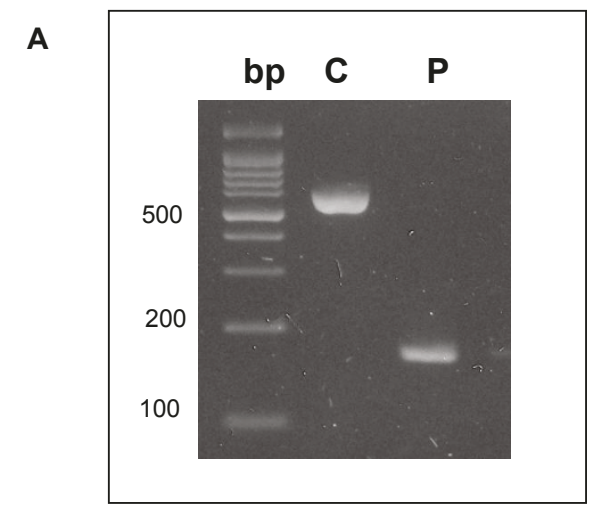

B

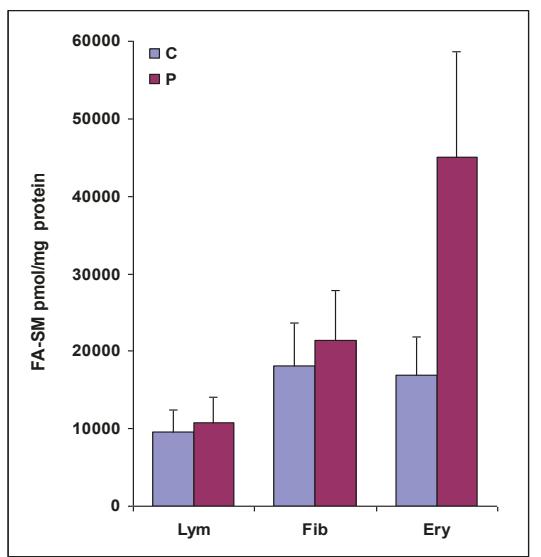

C

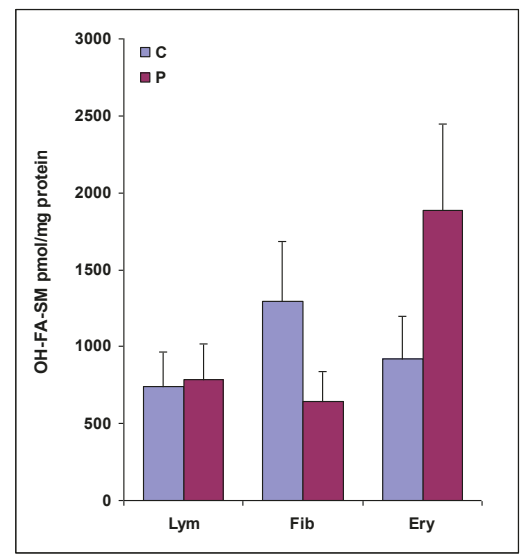

D

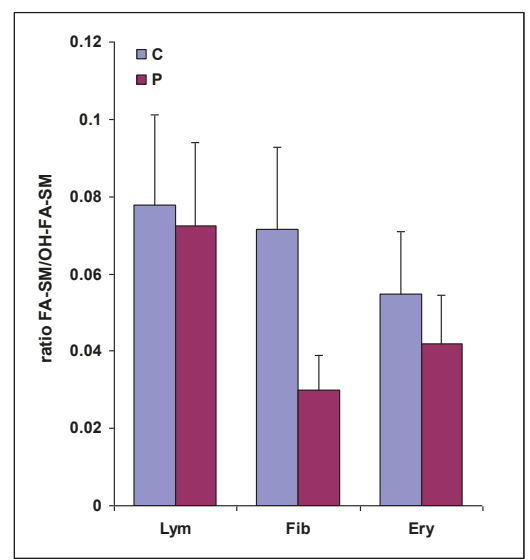

Figure 1 Lymphocyte FA2H patient transcript and total FA-SM/OH-SM profiles. A, Lane C shows the transcript obtained in control lymphocytes using the FA2H primer and lane P shows the shorter transcript obtained in patient lymphocytes. B, Total level of FA-SM in control and patient fibroblasts, lymphocytes and erythrocytes was quantitated by HPLC/MS-MS. C, Total level of OHFA-SM in control and patient fibroblasts, lymphocytes and erythrocytes was quantitated by HPLC/MS-MS. D, The ratio of the total OHFA-SM content divided by the total FASM content was calculated (mean of 2 experiments $+/-S D$ ).

C20 is the most hydroxylated saturated fatty acid in both control and patient, whereas C20:1 is the most hydroxylated monounsaturated acid in the controls and C24:1 the most hydroxylated monounsaturated acid in the patient. In erthrocytes, the ratio is decreased for the patient vs the control for all chain lengths, most noticeably for $\mathrm{C} 20$ and $\mathrm{C} 22$, with $\mathrm{C} 20$ and C24:1 the most heavily hydroxylated acids.

As SM is the major sphingolipid in human cultured fibroblasts [16], and taking into account the limited amount of sample, we focused our studies on this sphingolipid in cells obtained from patients using minimally invasive sampling. The lack of difference in the SM fatty acid and hydroxy fatty acid profile between patient and control lymphocytes was unexpected. Apparently the lack of FA2H as demonstrated by RT-PCR had no effect. On the other hand, SM hydroxy fatty acid content in fibroblasts was clearly decreased, with respect to both the total content and the fraction of hydroxylated
SM. The high OHFA content but low OHFA-SM/FASM ratio in erythrocytes suggests an imbalance, rather than a quantitative change. The distribution of OHFA according to chain length is different in the patient and control fibroblasts, with C20:1 being the most hydroxylated acid in the controls and C24:1 the most hydroxylated in the patient.

The results we obtained for FA content and distribution confirm previous results [16] however in that paper the authors did not find any hydroxy fatty acids. They conclude that if present, they must be in amounts below their detection limit of a few pmoles. This is certainly not in agreement with the results presented here. The discrepancy could be due to differences in the sample preparation as well as analytical conditions.

The characterization of the FA and OHFA profiles was carried out also to identify differences between controls and patients which could possibly form the basis for a 


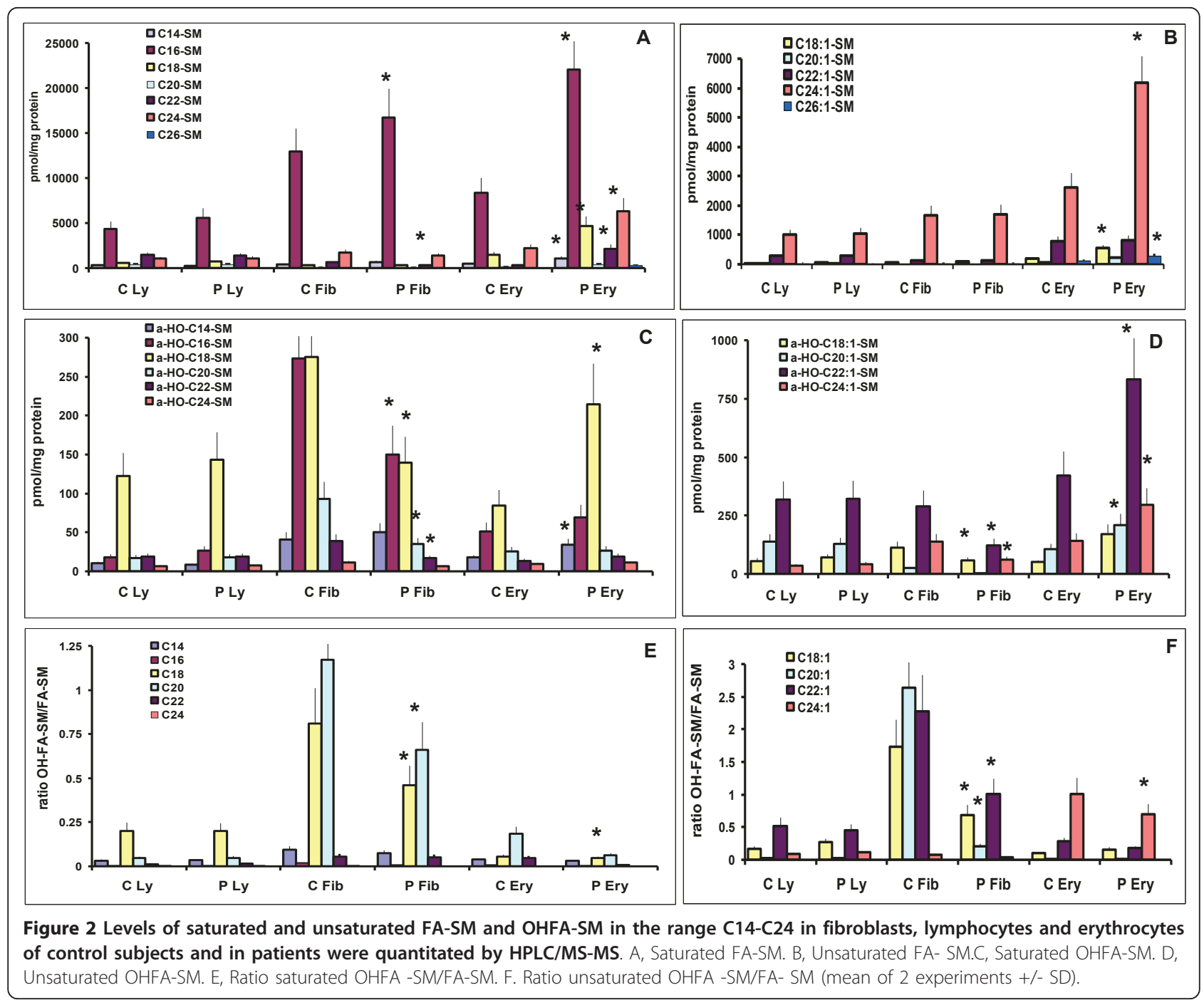

biochemical diagnostic test. The most significant difference was found in the relative decrease in OHFA-SM relative to FA-SM content in patient erythrocytes vs control. In cases where a skin biopsy is performed and fibroblasts are available, the decrease in saturated OHFA content coupled with the favoring of OHC24:1 in patients vs OHC20:1 in controls could also serve as a marker. Although genetic analysis is more feasible, FA-SM analysis could complement the molecular data and provide valuable information where genetic analysis is uninformative.

It is somewhat surprising that patient cells still contain a measurable amount of hydroxy fatty acids at all, considering the deleterious nature of the mutation in the FA2H gene. This would suggest presence of other enzyme/s with overlapping substrate specificity with FA2H. In fibroblasts and erythrocytes, which show a difference between the patient and the control, the 2-hydroxylating activity may be due to FA2H and to an additional enzyme with a different chain length preference. In lymphocytes, FA2H may be absent altogether while the proposed other enzyme performs the FA2-hydroxylation. Notably we were unable to detect any FA2H enzymatic activity in normal lymphocytes (results not shown).

\section{Conclusions}

We conclude that OHFA-SM is decreased in patient fibroblasts and erythrocytes. Differences in FA-SM and $\mathrm{OH}-\mathrm{SM}$ between patients and controls could serve as the basis for a diagnostic test.

\section{List of abbreviations}

FA2H: Fatty acid 2-hydroxylase; FA: Sphingomyelin:SM, Fatty acid; OHFA: Hydroxy fatty acids; GalC: galactosylceramide.

\section{Acknowledgements}

Dr. Chaya Miller and Mrs Sarah Weissman are acknowledged for technical assistance. 
This work was financed by The Legacy Heritage Biomedical Science Partnership Program of the Israel Science Foundation grant \# 1395/09

\section{Author details}

1Department of Genetics and Metabolic Diseases and the Monique and Jacques Roboh Department of Genetic Research, Hadassah-Hebrew University Medical Center, Jerusalem, Israel. ${ }^{2}$ Pediatric Neurology Unit Hadassah-Hebrew University Medical Center, Jerusalem, Israel. ${ }^{3}$ Department of Biochemistry and Molecular Biology, Medical University of South Carolina, Charleston SC, USA.

\section{Authors' contributions}

$A R$ and PD prepared the samples, interpreted the results and prepared the manuscript, SE cared for the patients, $\mathrm{HH}$ and JB were responsible for the lipid analysis. All authors have read and approved the final manuscript.

\section{Competing interests}

The authors declare that they have no competing interests.

Received: 11 April 2011 Accepted: 20 May 2011 Published: 20 May 2011

\section{References}

1. Morel P, Quarles R, Norton W: Basic Neurochemistry. New York: LippincottRaven; 6 1999, 69-94.

2. Kishimoto $Y$, Radin NS: Occurrence of 2-hydroxy fatty acids in animal tissues. J Lipid Res 1963, 4:139-143.

3. Raghavan S, Kanfer JN: Ceramide galactoside of enriched neuronal and glial fractions from rat brain. J Biol Chem 1972, 247:1055-1056.

4. Boggs JM, Koshy KM, Rangaraj G: Influence of structural modifications on the phase behavior of semi-synthetic cerebroside sulfate. Biochim Biophys Acta 1988, 938:361-372

5. Hama H: Fatty acid 2-Hydroxylation in mammalian sphingolipid biology. Biochim Biophys Acta 2010, 1801:405-415.

6. Alderson NL, Rembiesa BM, Walla MD, Bielawska A, Bielawski J, Hama H: The human FA2H gene encodes a fatty acid 2-hydroxylase. J Biol Chem 2004, 279:48562-48568

7. Uchida $Y$, Hama H, Alderson NL, Douangpanya S, Wang $Y$, Crumrine DA Elias PM, Holleran WM: Fatty acid 2-hydroxylase, encoded by FA2H, accounts for differentiation-associated increase in 2-OH ceramides during keratinocyte differentiation. J Biol Chem 2007, 282:13211-13219.

8. Zöller I, Meixner M, Hartmann D, Büssow H, Meyer R, Gieselmann V, Eckhardt M: Absence of 2-hydroxylated sphingolipids is compatible with normal neural development but causes late-onset axon and myelin sheath degeneration. J Neurosci 2008, 28:9741-9754.

9. Potter KA, Kern MJ, Fullbright G, Bielawski J, Scherer SS, Yum S, Li J, Cheng H, Han X, Kummetha-Venkata J, Khan Pathan AA, Rohrer B, Hama H: Central nervous system dysfunction in a mouse model of $\mathrm{FA} 2 \mathrm{H}$ deficiency. Glia 2011.

10. Edvardson S, Hama H, Shaag A, Gomori JM, Berger I, Soffer D, Korman SH, Taustein I, Saada A, Elpeleg O: Mutations in the fatty acid 2-hydroxylase gene are associated with leukodystrophy with spastic paraparesis and dystonia. Am J Hum Genet 2008, 83:643-648.

11. Dick KJ, Eckhardt M, Paisán-Ruiz C, Alshehhi AA, Proukakis C, Sibtain NA Maier H, Sharifi R, Patton MA, Bashir W, Koul R, Raeburn S, Gieselmann V,

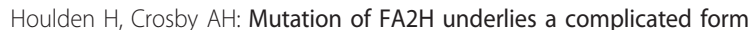
of hereditary spastic paraplegia (SPG35). Hum Mutat 2010, 31:E1251-1260.

12. Kruer MC, Paisán-Ruiz C, Boddaert N, Yoon MY, Hama H, Gregory A, Malandrini A, Woltjer RL, Munnich A, Gobin S, Polster BJ, Palmeri S, Edvardson S, Hardy J, Houlden H, Hayflick SJ: Defective FA2H leads to a novel form of neurodegeneration with brain iron accumulation (NBIA). Ann Neurol 2011, 68:611-618.

13. Bielawski J, Szulc ZM, Hannun YA, Bielawska A: Simultaneous quantitative analysis of bioactive sphingolipids by high-performance liquid chromatography-tandem mass spectrometry. Methods 2006, 39:82-91.

14. Bielawski J, Pierce JS, Snider J, Rembiesa B, Szulc ZM, Bielawska A: Comprehensive quantitative analysis of bioactive sphingolipids by highperformance liquid chromatography-tandem mass spectrometry. Methods Mol Biol 2009, 579:443-467.

15. Lowry OH, Rosebrough NJ, Farr AL, Randall RJ: Protein measurement with the Folin phenol reagent. J Biol Chem 1951, 193:265-275.
16. Valsecchi M, Mauri L, Casellato R, Prioni S, Loberto N, Prinetti A, Chigorno V, Sonnino S: Ceramide and sphingomyelin species of fibroblasts and neurons in culture. J Lipid Res 2007, 48:417-424.

doi:10.1186/1476-511X-10-84

Cite this article as: Dan et al:: 2-hydroxylated sphingomyelin profiles in cells from patients with mutated fatty acid 2-hydroxylase. Lipids in Health and Disease 2011 10:84.

\section{Submit your next manuscript to BioMed Central and take full advantage of:}

- Convenient online submission

- Thorough peer review

- No space constraints or color figure charges

- Immediate publication on acceptance

- Inclusion in PubMed, CAS, Scopus and Google Scholar

- Research which is freely available for redistribution

Submit your manuscript at www.biomedcentral.com/submit
C) Biomed Central 